Thorax (1968), 23, 640.

\title{
An unusual aortic coarctation
}

\author{
B. T. LE ROUX AND M. A. WILLIAMS \\ From the Thoracic Unit, University of Natal, Durban, South Africa
}

An unusual variety of aortic coarctation is described in which the left common carotid, left subclavian, and aberrant right subclavian arteries arose distal to the coarcted segment. The literature relating to unusual coarctation is briefly reviewed.

From the time that coarctation of the aorta was first described by Morgagni in 1771 (cited by Evans, 1933) until 1944, when Crafoord and Nylin (1945) and Gross (1945) independently managed the lesion successfully by a surgical procedure, the lesion remained a curiosity which challenged the descriptive powers of anatomists and pathologists and which proved a diagnostic exercise for clinicians. The literature during that period was confined to descriptions of the clinical and pathological features of the disease and its natural course, and to statistical evaluation of its various facets. Bonnet (1903) offered a classification which recognized an infantile and an adult type of coarctation. Fawcett (1905), Blackford (1928), and Evans (1933) recorded the necropsy incidence of the disease, which varied from 0.6 to $0.14 \%$. A constant finding in these series was that males were affected twice as commonly as females. In an analysis of 200 necropsy cases, Abbott (1928) recorded that only 28 had been diagnosed in life.

Bonnet's original classification has been modified by Hudson (1965), who relates infantile coarctation to a persistent ductus arteriosus, recognizes pre-ductal and post-ductal varieties, and includes an extreme form in which the whole ascending aorta is involved. This last-mentioned variety is a form of the hypoplastic left heart syndrome and is generally incompatible with life. Adult coarctation is classified in relation to the subclavian artery, and the usual type occurs beyond the origin of the left subclavian artery. Coarctation at or proximal to the left subclavian artery is uncommon. Bayley and Holoubek (1940) described a case of coarctation proximal to the left subclavian artery and were able to compare this with only 15 previously recorded examples. Since their description a number of cases of coarctation at this level have been described. In Fig. 1 are illustrated the varieties of infantile and adult coarctation, including the last-mentioned anomaly.
The rare varieties of coarctation previously reported are illustrated in Figure 2. Fawcett (1905) described coarctation of the aorta distal to the left subclavian artery in a patient in whom there was an aberrant right subclavian artery which arose proximal to the coarctation (Fig. 2a). Similar anomalies have since been described by East (1932) and by DeBoer, Grana, Potts, and Lev (1961). Gross (1950) quotes a case described by Stephens in which an aberrant right subclavian artery arose distal to the site of aortic coarctation (Fig. 2b). This anomaly has been recorded by others (Griffith, Oblath, and Jones, 1955 ; English, 1965). An anomalous right subclavian artery which arose at the site of the coarctation and was associated with a left innominate artery proximal to the coarctation was described by Partyka et al. (1958) (Fig. 2c). Aortic coarctation proximal to both the left subclavian artery and an anomalous right subclavian artery was first described by McGregor and Medalie (1952) (Fig. 2d). Since then D'Abreu, Aldridge, Astley, and Jones, (1961), Dodrill and Benson (1962), and Nathoo (1965) have described similar cases. That described by $\mathbf{M c G r e g o r}$ and Medalie (1952) had an associated small persistent ductus arteriosus. In the same year Brynolf, Crafoord, and Mannheimer (1958) recorded an example of coarctation of the aorta with a right aortic arch and a left descending aorta with the coarctation proximal to both subclavian arteries (Fig. 2e). Efskind and Sanderud (1955) described a patient with a double aortic coarctation, the coarcted segments being proximal and distal to a vessel from which left subclavian and left common carotid arteries arose (Fig. 2f). Clarke and Dodrill (1952) described a 'functional' coarctation; a single vessel was the source of both common carotid arteries and the right subclavian artery (itself a not uncommon anomaly), and during systole the distal lip of the origin of the left subclavian artery was displaced caudally and produced the syndrome of aortic coarctation (Fig. 3). 


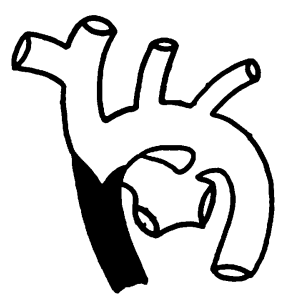

a

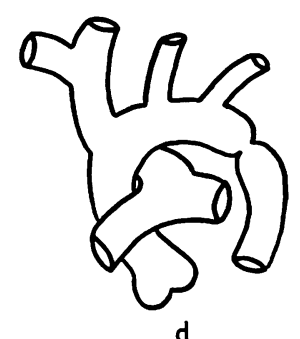

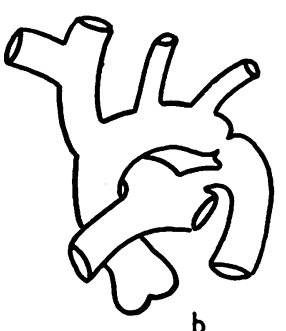

b

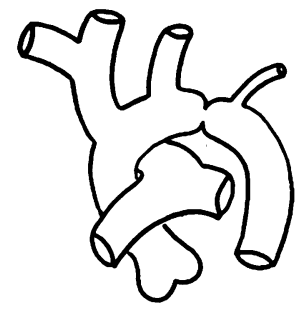

e

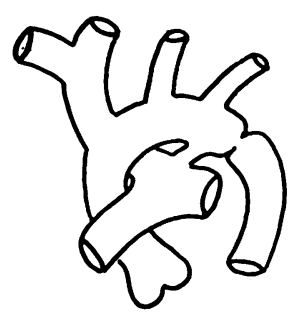

c

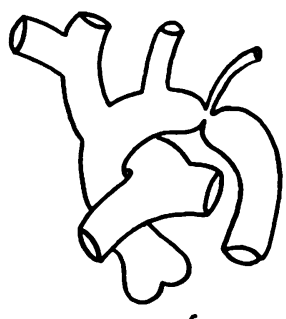

$f$

FIG. 1. (a) Atresia of ascending aorta; (b) pre-ductal infantile coarctation; (c) postductal infantile coarctation; (d) adult coarctation distal to left subclavian artery; (e) adult coarctation proximal to left subclavian artery; (f) adult coarctation at the left subclavian artery.
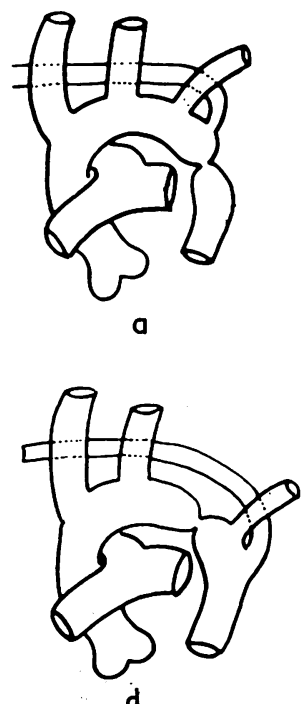

d

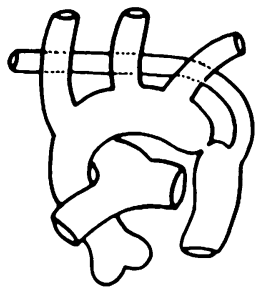

b

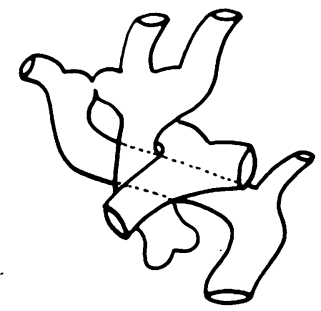

e
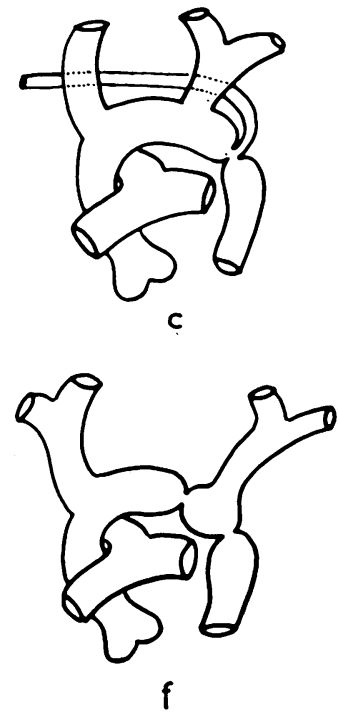

FIG. 2. (a) Aberrant right subclavian artery proximal to coarctation; (b) aberrant right subclavian artery distal to coarctation; (c) aberrant right subclavian artery arising at coarctation with left innominate artery; (d) left and aberrant right subclavian arteries distal to coarctation; (e) right aortic arch, left descending aorta, and coarctation proximal to both subclavian arteries; (f) double coarctation excluding left innominate artery. 


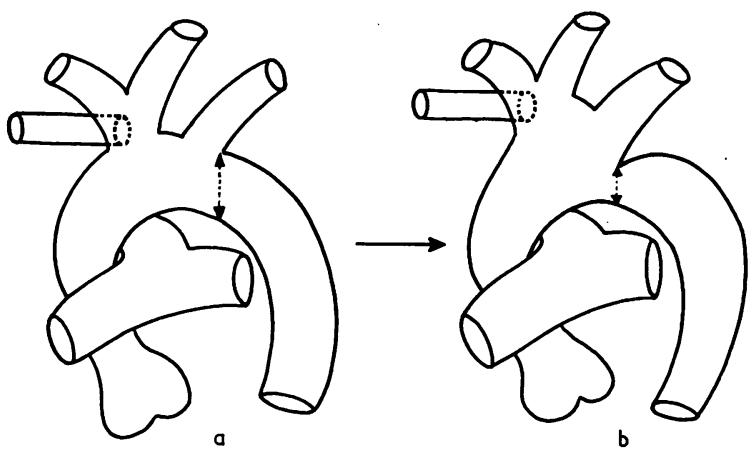

FIG. 3. 'Functional' coarctation, described in text.

There follows a description of an unusual coarctation to which reference has not been found in the literature.

\section{CASE REPORT}

An 18-year-old boy was admitted in 1966 to the Thoracic Surgical Unit, in Durban, for the management of aortic coarctation. The diagnosis of aortic coarctation had been made shortly after birth (he was the son of a medical practitioner), but the child remained well and an indication for surgical intervention was thought not to exist. Referral for surgical management was prompted by the patient's complaint of an uncomfortable pulsation in the neck. The boy was tall and well built. There was a thoracic scoliosis, convex to the right. Neither radial pulse was palpable ; the blood pressure in the right arm was $80 / 70 \mathrm{~mm}$. $\mathrm{Hg}$ and that in the left $70 / 60 \mathrm{~mm}$. Hg. Femoral and pedal pulses were impalpable and the blood pressure in the lower limbs could not be recorded. The striking clinical feature was the visible pulsation of the right carotid artery in the neck. The left carotid artery was not palpable. There was a systolic murmur over the praecordium, maximal at the third left interspace.

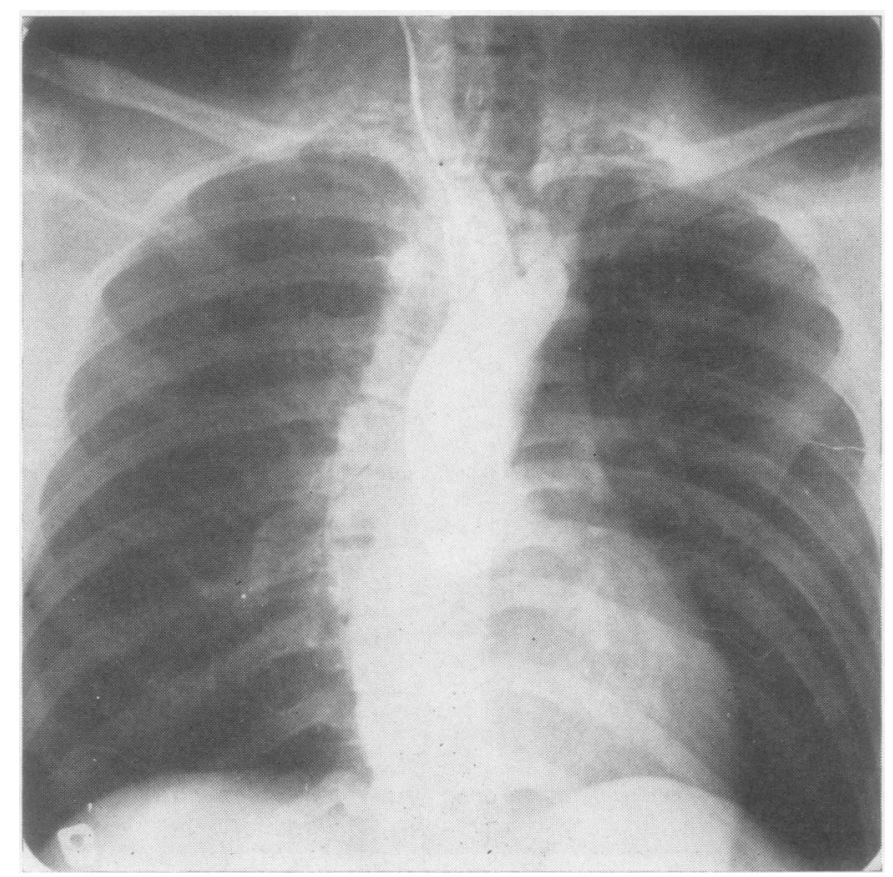

FIG. 4. Injection of contrast proximal to coarctation showing only right common carotid artery and ascending aorta. 


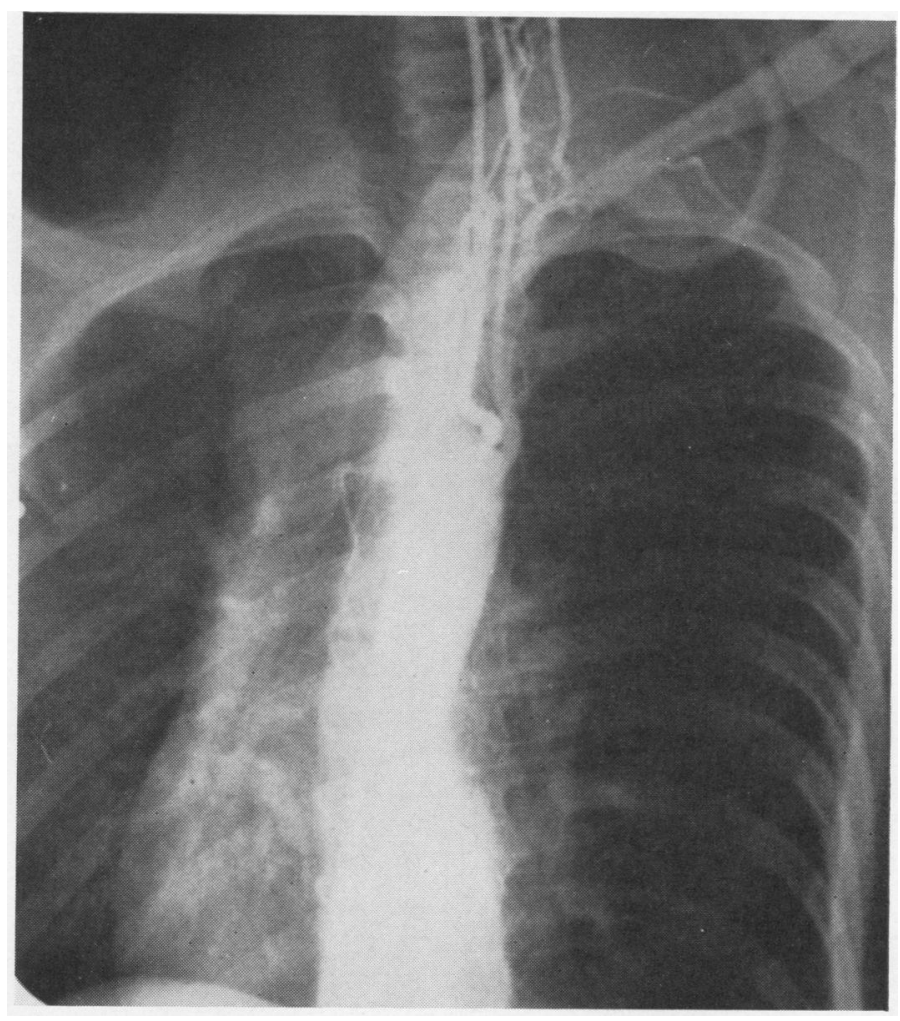

FIG. 5. Injection of contrast distal to coarctation showing left subclavian and carotid arteries.

Anastomotic channels about the scapulae were not appreciated and a chest radiograph did not show rib notching.

The ascending aorta was demonstrated radiographically by the injection of contrast through a catheter introduced through the right carotid artery. The right carotid artery was shown to be of a diameter nearly equal to that of the ascending aorta, of which it was virtually an extension to the neck; other aortic branches were not demonstrated by this injection (Fig. 4). The aorta and its branches distal to the coarctation were delineated by retrograde catheterization of the right femoral artery. The distal aorta, left carotid and left subclavian arteries were clearly demonstrated (Fig. 5). Absence of the right radial pulse made reasonably certain the existence of an aberrant right subclavian artery, but this was not demonstrated by contrast studies.

Through a left lateral thoracotomy and with controlled hypotension (trimetaphan (Arfonad)) the coarctation was exposed. The strikingly large right common carotid artery was identified, and immediately distal to this was a vessel which divided shortly after its origin and was judged to be the thyroidea ima artery. There followed the coarctation, beyond which the slender left common carotid and subclavian vessels were identified. Yet more distally the right subclavian artery passed posteriorly and medially behind the oesophagus. There was an aneurysm on the inferior aspect of the post-stenotic dilatation of the aorta, one aspect of which was so thin that blood could be seen swirling within it. The aorta was clamped central to the coarctation, distal to the right common carotid artery, and at the level of the aberrant right subclavian artery; the left subclavian and left common carotid arteries were controlled; the area of aorta excluded by clamps was incised in its length across the coarctation, and the aneurysm was resected. A diaphragm which occluded the aortic lumen was excised and a gusset of woven Dacron was used to replace the defect-the 'isthmusplastic' operation of Vossschulte (1961). Pulsation and pressure in the distal aorta, after removal of the clamps, were not different from that proximal to the site of aortic repair. Convalescence was uneventful ; the patient was transiently hoarse, and it was presumed that this was the consequence of trauma to the left recurrent laryngeal nerve, which had been identified at operation where it looped under the aorta at the site of the coarctation and on which traction had been considerable. The boy's voice was normal before discharge. All peripheral pulses became easily palpable after operation and right carotid pulsation 


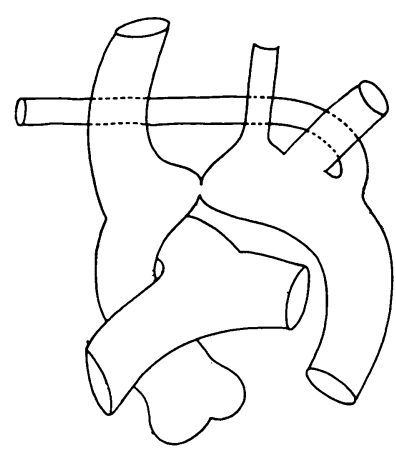

FIG. 6. Coarctation proximal to left common carotid and left and aberrant right subclavian arteries.

was markedly reduced. The boy remains well. The coarctation is shown diagramatically in Fig. 6, and an artist's impression of the pre- and post-operative state is shown in Figure 7 ( $a$ and $b$ ).

\section{REFERENCES}

Abbott, M. E. (1928). Coarctation of the aorta of the adult type. Amer. Heart J., 3, 392, 574.

Bayley, R. H., and Holoubek, J. E. (1940). Coarctation of the aorta at or above the origin of the left subclavian artery. Brit. Heart $J$., $2,208$.

Blackford, L. M. (1928). Coarctation of the aorta. Arch. intern. Med., 41, 702 .

Bonnet, L. M. (1903). Sur la lesion dite sténose congénitale de l'aorte dans la region de l'isthme. Rev. Méd. (Paris), 23, 108.

Brynolf, I., Crafoord, C., and Mannheimer, E. (1958). Coarctation of the aorta proximal to both subclavian arteries. J. thorac. Surg., 35,123 .

Clarke, N. E., and Dodrill, F. D. (1952). Coarctation of aorta. Amer. Heart J., 43, 108.

Crafoord C., and Nylin, G. (1945). Congenital coarctation of the aorta and its surgical treatment. J. thorac. Surg., 14, 347.

DeBoer, A., Grana, L., Potts, W. J., and Lev, M. (1961). Coarctation of the aorta. Arch. Surg., 82, 801.

D'Abreu, A. L., Aldridge, A. G. V., Astley, R., and Jones, M. A. C. (1961). Coarctation of the aorta proximal to both subclavian arteries producing reversible papilloedema. Brit. J. Surg., 48, 525.

Dodrill, F. D., and Benson, C. D. (1962). Coarctation of the aorta with both subclavian arteries arising from the distal segment complicated by postoperative intussusception. Surgery, 51, 809.

East, T. (1932). Coarctation of the aorta. Proc. roy. Soc. Med., 25, 796.

Efskind, L., and Sanderud, A. (1955). An unusual case of coarctation of the aorta. J. thorac. Surg., 29, 665.

English, T. A. H. (1965). Direct left ventriculofemoral bypass during resection of coarctation of the aorta with anomalous subclavian arteries. Thorax, 20, 36.

Evans, W. (1933). Congenital stenosis (coarctation) atresia, and interruption of the aortic arch. Quart. J. Med., N.S. 2, 1.

Fawcett, J. (1905). Coarctation of the aorta as illustrated by cases from the post-mortem records of Guy's Hospital from 1826-1902. Guy's Hosp. Rep., 59, 1.

Griffith, G. C., Oblath, R. W., and Jones, J. C. (1955). Unusual manifestations of coarctation of the aorta. Circulation, 12, 1080.

Gross, R. E. (1945). Surgical correction for coarctation of the aorta. Surgery, 18, 673.

- (1950). Coarctation of the aorta. Surgical treatment of 100 cases. Circulation, $1,41$.

Hudson, R. E. B. (1965). Cardiovascular Pathology. Arnold, London.

McGregor, M., and Medalie, M. (1952). Coarctation of the aorta Brit. Heart J., 14, 531.

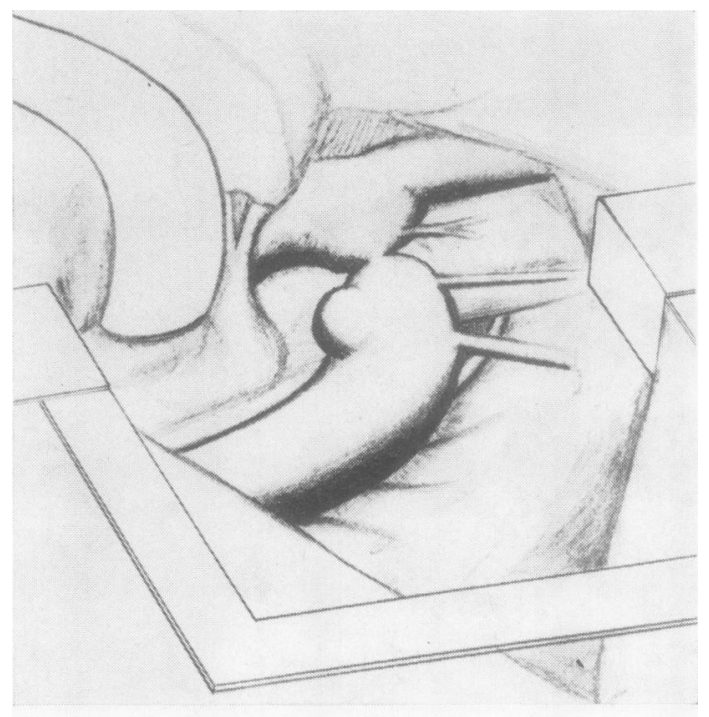

(a)

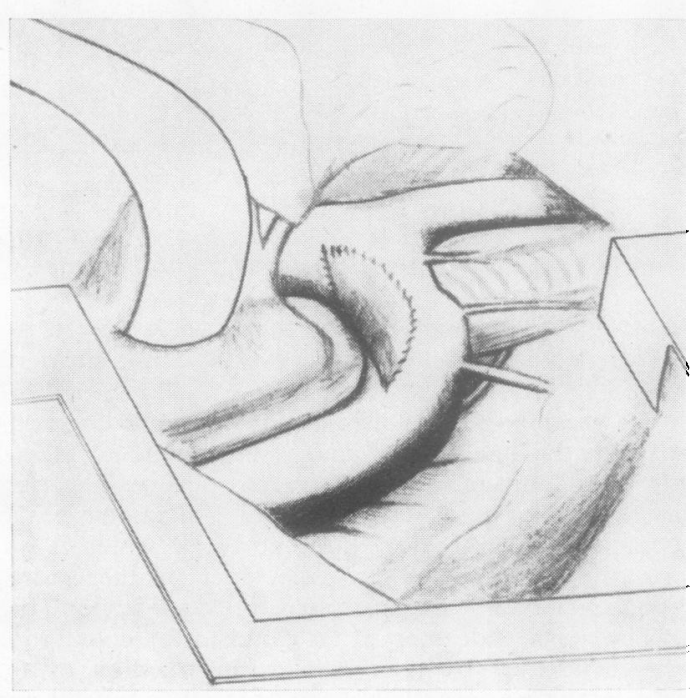

(b)

FIG. 7. (a and b) Artist's impression of pre- and postoperative states.

Nathoo, A. R. (1965). Two unusual cases of coarctation of the aorta. Guy's Hosp. Rep., 114, 32.

Partyka, L. C., French, S. W., and Rumer, G. F. (1958). Anomalous innominate and right subclavian arteries associated with coarctation of aorta. Arch. Surg., 77, 81.

Vossschulte, K. (1961). Surgical correction of coarctation of the aorta by an "isthmusplastic" operation. Thorax, 16, 338. 